\title{
REVIEW OF NUMERICAL SCHEMES AND BOUNDARY CONDITIONS APPLIED TO WAVE PROPAGATION PROBLEMS
}

\author{
O. de Almeida \\ Universidade Federal de Uberlândia \\ Departamento de Engenharia Mecânica \\ FEMEC \\ Campus Santa Mônica \\ CP. 38400-902, Uberlândia, MG, Brasil \\ odenir.almeida@ufu.br \\ Received: February 13, 2016 \\ Revised: March 18, 2016 \\ Accepted: May 06,2016
}

\section{ABSTRACT}

As a review framework, the present study describes the application and performance of different numerical schemes for Computational Aeroacoustics (CAA) of simple wave propagation problems. The current approach aims to simulate pulse propagation on the near field by the use of different spatial and temporal numerical schemes for the full and Linearized Euler Equations (LEE) in a dimensional and dimensionless formulation. Comparisons of processing time, residual error and quality of results are present and discussed shedding light to the relevant parameters which play important role in aeroacoustics. The investigation is focused on different Gaussian pulse propagation cases in unbounded and bounded domains which is solved by using optimized spatial and temporal schemes for reducing dissipative and dispersive errors. The numerical results are compared with the exact analytical solutions when available, showing good agreement.

Keywords: wave propagation, numerical schemes, low dissipation, low dispersion, aeroacoustics

\section{NOMENCLATURE}

M Mach number

DRP Dispersion-relating-preserving

FDo Finite difference scheme

D thickness, $m$

$\mathrm{J} \quad$ Bessel functions

p dimensionless pressure

$\mathrm{u}_{1}, \mathrm{u}_{2}, \mathrm{u}_{3}$ velocity components, $\mathrm{m} / \mathrm{s}$

$\mathrm{x}, \mathrm{y}, \mathrm{z}$ cartesian coordinates, $\mathrm{m}$

\section{Greek symbols}

$\varepsilon \quad$ pressure pulse amplitude

$\theta \quad$ angular coordinate inside domain

$\rho$ density, $\mathrm{kg} / \mathrm{m}^{3}$

$\Delta \mathrm{t} \quad$ time-step

\section{Subscripts}

0 base flow

$\mathrm{x}, \mathrm{y} \quad$ cartesian coordinates (direction)

RMS root mean square

$\infty \quad$ free stream

\section{INTRODUCTION}

The fundamental of noise generated by aerodynamic flow has been examined extensively by theoretical and experimental studies along the last century and more recently has evolved intensively in the computational field being originally called Computational Aero-Acoustics (CAA). In summary, the main physical idea behind CAA is the requirement of preserving the shape and frequency of wave generation and propagation. Numerically, this statement translates to the need of numerical schemes suited to handle multiple scales, including long and short waves and long-time integration with minimal dissipation and dispersion errors.

With these restrictions imposed, classical Computational Fluid Dynamics (CFD) cannot guarantee a precise wave solution, since the codes are dispersive and dissipative, due to the low order numerical schemes employed for solving the governing equations. A way to overcome this problem is to construct spatial and temporal schemes optimizing the finite difference approximations in the wave number and frequency space. This approach will assure that the numerical solutions of a high order finite difference scheme will have the same number of wave's modes (acoustic, entropy and vorticity waves).

Table 1 presents a review of numerical schemes, proposed in the literature, applied exclusively to wave propagation problems. There are a lot of others schemes which follow the idea of optimization in the Fourier space (spectral resolution) - Lele (1992), Lockard et al. (1995), Haras and Ta'asan (1994) among others. These schemes are frequently used for shock noise prediction, scattering of acoustic pulses, subsonic flow past over obstacles and jets.

The present paper is devoted to a review of numerical schemes applicable for the propagation of acoustic waves throughout a $2 \mathrm{D}$ (two-dimensional) computational domain. The main focus was given to the direct application of already known numerical techniques for such class of problem. In addition, numerical investigation and comparison of different 
parameters intrinsically associated with the numerical implementation such as spatial (Tam and Webb, 1993) and temporal discretizations Willianson (1980), Hu et al. (1996) and Colonius et al. (1993) have been addressed. Global parameters like processing time and level of reflection were also evaluated during the test cases. Finally, to accomplish the task of evacuating the acoustic pulses through the boundaries of the computational domain, a set of boundaries conditions also derived for acoustics were applied. The well-known Radiation and Outflow as well as Perfectly Matched Layer (PML) boundary conditions (Hu, 1996a) were implemented and tested. The propagation and evacuation of the acoustic pulse is finally validated against the analytical solution for Linearized Euler Equations (LEE).

Table 1. Numerical schemes applied to wave propagation problems.

\begin{tabular}{|c|c|c|}
\hline \multicolumn{2}{|l|}{ Spatial Discretization } & \multirow{2}{*}{$\begin{array}{c}\text { References } \\
\text { Tam (1995), } \\
\text { Tam (1997), } \\
\text { Tam (2004) }\end{array}$} \\
\hline $\begin{array}{l}\text { Dispersion-Relating- } \\
\text { Preserving (DRP) }\end{array}$ & $\begin{array}{l}\text { In this scheme a central difference is } \\
\text { employed to approximate first derivatives. } \\
\text { The coefficients are optimized based on a } \\
\text { minimalisation of the dispersion error. }\end{array}$ & \\
\hline $\begin{array}{l}\text { Finite Difference } \\
\text { Optimized (FDo) }\end{array}$ & $\begin{array}{l}\text { Optimized scheme based on similar } \\
\text { approach for space discretization. The } \\
\text { difference is that the error is minimized } \\
\text { taking into account logarithm of the } \\
\text { wavenumber. The stability and accuracy } \\
\text { increase for these schemes. }\end{array}$ & $\begin{array}{c}\text { Bogey and } \\
\text { Bailly (2002), Zing } \\
\text { et al. (1993) }\end{array}$ \\
\hline \multicolumn{2}{|l|}{ Temporal Discretization } & References \\
\hline RK3 & $\begin{array}{l}\text { This scheme is only optimized for criteria of } \\
\text { low-storage. Previous use by the authors } \\
\text { showed good properties. }\end{array}$ & Berland et al.(2004) \\
\hline LDDRK5 & $\begin{array}{l}\text { Traditionally, the coefficients of the Runge- } \\
\text { Kutta scheme are optimized to minimize } \\
\text { the dissipation and propagation waves. The } \\
\text { optimization does not compromise the } \\
\text { stability consideration. }\end{array}$ & $\begin{array}{c}\text { Stanescu and } \\
\text { Habashi (1998), } \\
\text { Berland et al.(2005), } \\
\text { Colonius et } \\
\text { al.(1993) } \\
\end{array}$ \\
\hline RKO5 & $\begin{array}{l}\text { Optimized scheme based on similar } \\
\text { approach for space discretization. The } \\
\text { difference is that the error is minimized } \\
\text { taking into account logarithm of the } \\
\text { wavenumber. The stability and accuracy } \\
\text { increase for these schemes. }\end{array}$ & Roeck et al.(2004) \\
\hline \multicolumn{2}{|l|}{ Boundary Treatment } & References \\
\hline Buffer Zones & $\begin{array}{l}\text { Introduction of additional zones of grid } \\
\text { points to surround the truncated domain so } \\
\text { that the disturbances are attenuated. }\end{array}$ & $\begin{array}{l}\text { Colonius et al. } \\
\text { (1993), Hu and } \\
\text { Atkins(2002), } \\
\text { Ta'asan and } \\
\text { Nark(1995) } \\
\end{array}$ \\
\hline Radiation and Oufflow & $\begin{array}{l}\text { Based on asymptotic solution of Linearized } \\
\text { Euler Equations. These approaches are very } \\
\text { accurate as it looks for the direction of the } \\
\text { direction of the wave that is reaching the } \\
\text { boundaries of domain, and ensures that the } \\
\text { wave absorption is perpendicular to the } \\
\text { direction of wave propagation. }\end{array}$ & $\begin{array}{c}\text { Israeli and } \\
\text { Orszag(1981), } \\
\text { Giles(1990), } \\
\text { Givoli(1991), Tam } \\
\text { and Dong(1994), } \\
\text { Bayliss and } \\
\text { Turkel(1982) } \\
\end{array}$ \\
\hline $\begin{array}{l}\text { Perfectly Matched Layer } \\
\text { (PML) }\end{array}$ & $\begin{array}{l}\text { Absorbing layer that was originally } \\
\text { reflectionless for electromagnetic waves of } \\
\text { any frequency and direction. Adapted to } \\
\text { CAA. }\end{array}$ & 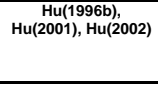 \\
\hline
\end{tabular}

\section{THEORY - GOVERNING EQUATIONS}

This section shows the equation set for the Linearized Euler Equations (LEE) in a dimensional and dimensionless formulation. Such set of equations has been suitable for wave propagation into a farfield - Tam and Webb (1993), Bogey and Bailly (2004) and Hu (1996a).

\section{A. The Linearized Euler Equation - Dimensional Formuale}

Acoustics wave propagation on a uniform mean flow is governed by Linearized Euler Equations (LEE) since for most of the aeroacoustics problems the Reynolds number based on wave length is high enough to make the viscous terms on Navier-Stokes negligible when compared with dynamic terms. The linearization of Euler equations is valid to predict wave propagation since acoustics waves involves small disturbances superimposed on a mean flow uniform by hypothesis. The use of Linearized Euler Equations is attractive because the relative small computational cost involved on simulations. Another advantage of this approach is its high capability on work with non-reflexive boundary conditions.

This approach is useful for academics cases, but, for commercial software and industrial applications this approach is not so interesting due its small generality. An important limitation of Linearized Euler equation is that this formulation cannot predict correctly physical problems when there is coupling between flow dynamics and acoustic generation and/or propagation.

The LEE for a 2D dimensional domain is presented as:

$$
\frac{\partial U}{\partial t}+\frac{\partial E}{\partial x}+\frac{\partial F}{\partial y}=H
$$

where:

$$
U=\left[\begin{array}{c}
\rho \\
u \\
v \\
p
\end{array}\right], E=\left[\begin{array}{c}
\rho_{0} u+\rho u_{0} \\
u_{0} u+p / \rho_{0} \\
u_{0} v \\
u_{0} p+p_{0} u
\end{array}\right], F=\left[\begin{array}{c}
\rho_{0} v \\
0 \\
p / \rho_{0} \\
\gamma p_{0} v
\end{array}\right]
$$

The no homogeneous term $H$ on the right side of Eq. (1) represent the distributed aeroacoustics sources. In this work these equations have been solved by applying DRP-schemes and using nonreflexive boundary treatment (see next section).

\section{B. The Linearized Euler Equation - Non dimensional Formuale}

The non-dimensional form of the Linearized Euler Equations is presented as:

$$
\frac{\partial u}{\partial t}+A \frac{\partial u}{\partial x}+B \frac{\partial u}{\partial y}=0
$$

where:

$$
u=\left(\begin{array}{l}
\rho \\
u \\
v \\
p
\end{array}\right), \quad A=\left(\begin{array}{cccc}
M & 1 & 0 & 0 \\
0 & M & 0 & 1 \\
0 & 0 & M & 0 \\
0 & 1 & 0 & M
\end{array}\right) \text {, }
$$




$$
B=\left(\begin{array}{llll}
0 & 0 & 1 & 0 \\
0 & 0 & 0 & 0 \\
0 & 0 & 0 & 1 \\
0 & 0 & 1 & 0
\end{array}\right),
$$

where the density, velocities, pressure and Mach number are written on its straight forward form. In this approach it's often assumed that the Mach number is always less than unity. This nondimensional formulation has been shown to introduce a specific technique for boundary condition treatment, which is presented in the next section.

\section{FORMULATION ON BOUNDARIES}

As stated by Tam and Webb (1993), in CAA numerical boundary conditions are often developed for idealized model problems. In practical applications, they must be modified or extended to account for the presence of a non-uniform and sometimes unknown mean flow. A reasonable boundary condition for CAA should be able to absorb all disturbances on a far-field without significant reflection of waves to inside of the computational domain. As a remark, this goal is very hard to be achieved by simple application of traditional CFD boundary conditions. Some interesting boundaries conditions are presented on literature. For Linearized Euler Equation the Perfectly Matched Layer of Hu (1996a) and the Radiation and Outflow Boundary condition of Tam and Webb (1993) seem to be the most powerful treatment on boundaries for aeroacoustics.

Briefly, the formulation for radiation and outflow as well as PML boundary condition are given on next sub-sections. For detailed information consult references listed on Table 1.

\section{A. The Radiation Boundary Condition}

Essentially, Radiation boundary conditions were based on the farfield asymptotic solutions for the Linearized Euler Equations, as presented by investigators like Bayliss and Turkel (1982) and summarized in Tam and Webb (1993). These boundary conditions will apply at the boundaries where there are only outgoing acoustic waves. In Cartesian coordinates the equations are:

$$
\begin{gathered}
\frac{1}{V(\theta)} \frac{\partial \rho}{\partial t}+\cos \theta \frac{\partial \rho}{\partial x}+\sin \theta \frac{\partial \rho}{\partial y}+\frac{\rho}{2 r}=0 \\
\frac{1}{V(\theta)} \frac{\partial u}{\partial t}+\cos \theta \frac{\partial u}{\partial x}+\sin \theta \frac{\partial u}{\partial y}+\frac{u}{2 r}=0 \\
\frac{1}{V(\theta)} \frac{\partial v}{\partial t}+\cos \theta \frac{\partial v}{\partial x}+\sin \theta \frac{\partial v}{\partial y}+\frac{v}{2 r}=0
\end{gathered}
$$

$$
\frac{1}{V(\theta)} \frac{\partial p}{\partial t}+\cos \theta \frac{\partial p}{\partial x}+\sin \theta \frac{\partial p}{\partial y}+\frac{p}{2 r}=0
$$

where

$$
V(\theta)=a_{0}\left[M \cos (\theta)+\left(1-M^{2} \sin ^{2} \theta\right)^{1 / 2}\right\rfloor
$$

\section{B. The Outflow Boundary Condition}

On a general form, at the outflow region the outgoing disturbances consist of a combination of acoustics, entropy and vorticity waves. To handle this more general and physical situation Tam and Webb (1993) presents the outflow boundary condition. In a Cartesian coordinates frame the equations are:

$$
\begin{gathered}
\frac{\partial \rho}{\partial t}+u_{0} \frac{\partial \rho}{\partial x}=\frac{1}{a_{0}^{2}}\left(\frac{\partial p}{\partial t}+u_{0} \frac{\partial p}{\partial x}\right) \\
\frac{\partial u}{\partial t}+u_{0} \frac{\partial u}{\partial x}=-\frac{1}{\rho_{0}} \frac{\partial p}{\partial x} \\
\frac{\partial v}{\partial t}+u_{0} \frac{\partial v}{\partial x}=-\frac{1}{\rho_{0}} \frac{\partial p}{\partial y} \\
\frac{1}{V(\theta)} \frac{\partial p}{\partial t}+\cos \theta \frac{\partial p}{\partial x}+\sin \theta \frac{\partial p}{\partial y}+\frac{p}{2 r}=0
\end{gathered}
$$

The equations above apply at the boundaries where outgoing disturbances consist of a combination of acoustic, vorticity and entropy waves.

\section{The Perfectly Matched Layer (PML)}

Following Hu (2002) the PML equation for the 2-D Euler equation is written in the form:

$$
\begin{gathered}
\frac{\partial u}{\partial t}+A \frac{\partial u}{\partial x}+B \frac{\partial u}{\partial y}+\sigma_{y} A \frac{\partial q}{\partial x}+\sigma_{x} B \frac{\partial q}{\partial y}+ \\
+\left(\sigma_{x}+\sigma_{y}\right) u+\sigma_{x} \sigma_{y} q+ \\
+\frac{\sigma_{x} M}{1-M^{2}} A\left(u+\sigma_{y} q\right)=0
\end{gathered}
$$

where $\mathrm{q}$ is an auxiliary variable defined as:

$$
\frac{\partial q}{\partial t}=u
$$


and $\sigma_{x}$ and $\sigma_{y}$ are the absorption coefficients often taken to be power functions, for example:

$\sigma_{x}(x)=\sigma_{m}\left(1-M^{2}\right)\left|\frac{x-x_{l}}{D}\right|^{\beta}, \quad \sigma_{y}=\sigma_{m}\left|\frac{y-y_{l}}{D}\right|^{\beta}$

where $\mathrm{x}_{1}$ or $\mathrm{y}_{\mathrm{l}}$ denotes the location where the PML zone starts, and $\mathrm{D}$ is the thickness of the PML domain. In this work values of $\sigma_{m}=2$ and $\beta=2$ are used as standard for the computations. However, additional tests were performed changing the reference values of these parameters.

\section{NUMERICAL METHODS}

In order to check the validity and effectiveness of these schemes for wave propagation problems, three canonical pulse propagation examples will be reviewed. The numerical implementation was concentrated in the DRP 7-point, Tam and Webb (1993) and Tam (1995), respectively, and the 9-point optimized central difference scheme of Bogey and Bailly (2004). For time integration two RungeKutta schemes were selected based on independent criteria of low-storage Willianson (1980) and lowdispersion/dissipation Hu et al. (1996).

\section{A. The Runge Kutta time integration method}

In this paper, the low-storage and lowdispersion/dissipation properties are evaluated through the implementation of the following RungeKutta schemes:

- 3rd order low-storage Runge-Kutta scheme - Willianson (1980) - RK3W

- 5 stages low-dissipation and low-dispersion 2nd order Runge-Kutta scheme - Hu et al.(1996) - LLDRK5

- 5 stages 2nd order optimized Runge-Kutta scheme - Bogey and Bailly (2004) - RKO5

\section{B. The finite difference schemes}

Tam and Webb in 1993 presented a complete methodology for treating Linearized Euler Equations. The spatial discretization is done by using the lowdispersion and low-dissipation numerical schemes also known as DRP (Dispersion-relation-preserving). This scheme seems to be very suitable for the propagation of an acoustic pulse. The complete description of optimized spatial discretization for the Dispersion-Relation-Preserving (DRP) schemes is given in Tam and Webb (1993).

The coefficients of the 7-point central difference scheme (DRP) for approximating spatial derivatives used in this work are:

$\mathrm{a} 0=0$

$$
\begin{aligned}
& \mathrm{a} 1=-\mathrm{a} 1=0.77088238051822552 \\
& \mathrm{a} 2=-\mathrm{a} 2=-0.166705904414580469 \\
& \mathrm{a} 3=-\mathrm{a} 3=0.02084314277031176
\end{aligned}
$$

This scheme was applied to all internal points in the computational domain. However, due to the use of such a large stencil it gives rise to 3 ghost points at the boundary of the computational domain.

\section{PERFORMANCE OF METHODS}

In order to evaluate the solution accuracy for the simulations, the following residual error formulae has been employed:

$$
E_{R M S}=\sqrt{\sum_{j=1}^{N} \frac{\left(u_{\text {exact }}-u_{\text {numeric }}\right)^{2}}{N}},
$$

where $N$ is the number of points.

The exact solution was obtained solving the following set of equations as presented in Tam and Webb (1993).

$u(x, y, t)=\frac{\varepsilon_{1}(x-M t)}{2 \alpha_{1} \eta} \int_{0}^{\infty} e^{-\xi^{2} / 4 \alpha_{1}} \sin (\xi t) J_{1}(\xi \eta) \xi d \xi$

$v(x, y, t)=\frac{\varepsilon_{1} y}{2 \alpha_{1} \eta} \int_{0}^{\infty} e^{-\xi^{2} / 4 \alpha_{1}} \sin (\xi t) J_{1}(\xi \eta) \xi d \xi$

$p(x, y, t)=\rho=\frac{\varepsilon_{1}}{2 \alpha_{1}} \int_{0}^{\infty} e^{-\xi^{2} / 4 \alpha_{1}} \cos (\xi t) J_{0}(\xi \eta) d \xi$

where $\eta=\left[(x-M t)^{2}+y^{2}\right]^{1 / 2}, \quad \mathrm{~J}_{0}$ and $\mathrm{J}_{1}$ are respectively, the Bessel functions of first kind and zero and first order.

At the time $\mathrm{t}=0$ the initial condition is assumed to be $\mathrm{u}=\mathrm{v}=0$ and $\mathrm{p}$ is a Gaussian distribution. To advance the solution in time, the simulations have been run using different CFL numbers varying from $0.05,0.1$ and 0.5 . These values were selected based on numerical stability criteria presented by the DRP scheme. The maximum $\Delta \mathrm{t}$ for advancing the solution is given by the following criterion:

$\Delta t_{\max }=\frac{0.4}{1.75\left[M+\left(1+(\Delta x / \Delta y)^{2}\right)^{1 / 2}\right]} \frac{\Delta x}{a_{0}}$

in which, applying the current values of $M=0.5$, $\Delta x=\Delta y=1$ and $\mathrm{a} 0=1.0$ results in $\Delta t_{\max }=0.119$.

\section{TEST CASE - PULSE PROPAGATION}

Three different pulse propagation problems are investigated in this work. The first two problems are based on a set of Gaussian acoustic, entropy and 
vorticity pulses applied on a Cartesian unbounded 2D domain:

\section{A. Gaussian acoustic, entropy and vorticity waves - CASE (A)}

A Gaussian Acoustic Pulse of form of Equation (21) has been applied. At the time $t=0$ an acoustic pulse is generated at the center of the domain and then consecutively propagated through it until reaching the boundaries - Figure 1 .

Physically, this problem represents the propagation of an acoustic pulse in a medium at rest or subjected to free stream mean flow of Mach equal to 0.5. Based on that, two test conditions were analyzed based on the Mach number of the mean flow. In the case of mean flow an entropy pulse is released at the same time and at a distance of about $1 / 3$ of the length of the computational domain. This is done in order to force both perturbations to be at same time leaving the domain through the right boundary as they are propagated. The numerical results for such simulations were compared against each other and against the analytical solution for the LEE.

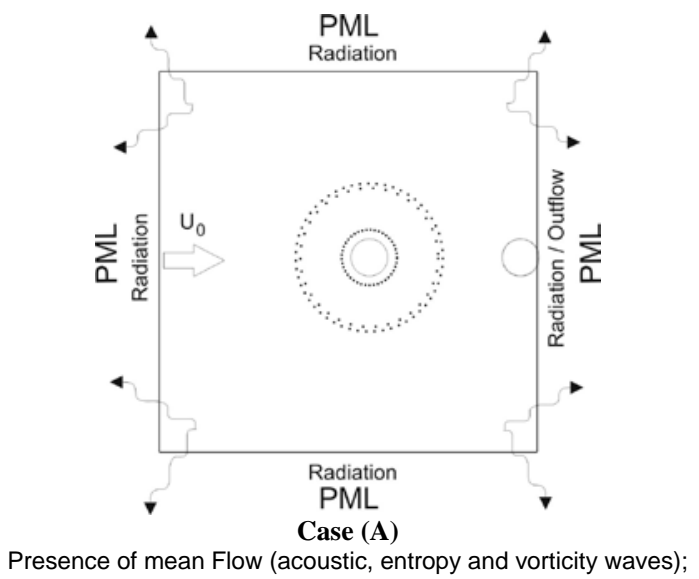

Figure 1. Illustration of wave propagation problems.

The pressure, entropy and vorticity pulses were given by a Gaussian distribution:

$f(x, y)=\varepsilon_{1} \exp \left[-\ln 2\left(\frac{x+y}{b}\right)^{2}\right]$

The following parameters were applied:

Pressure pulse amplitude $\varepsilon 1=0.01$

Half-width $b=3.0$

Entropy pulse amplitude $\varepsilon 2=0.001$

Half-width $b=5.0$

Vorticity pulse amplitude $\varepsilon 2=0.004$

Half-width $b=5.0$
In order to analyze the effect of reflection on boundaries an equal spaced, orthogonal mesh is adopted. The domain length is $-100 \leq x \leq 100$ and $-100 \leq y \leq 100$, and the domain has $201 \times 201$ grid points. This leads to a mesh spacing $\Delta x=\Delta y=1$. Such grid points have been applied to the three cases to be investigated in this work. To maintain numerical stability a Courant-FriedrichsLewy (CFL) number of 0.1 is used to the present problem simulations. As the non-dimensional sound velocity is adopted as 1 , on present problem, we can conclude that the time step is 0.1 .

\section{B. Acoustic pulse through a mesh stretching - CASE (B)}

In order to investigate the grid-spacing influence in a wave propagation problem, this case has been arbitrary selected. This case is a variation of Case (A) where only an acoustic pulse (following the same parameters) is emitted and convected through a 2D domain. At the position $x \geq+50$ the grid spacing is varied assuming different stretching rates of $5 \%, 10 \%$ and $50 \%$ - Figure 2.

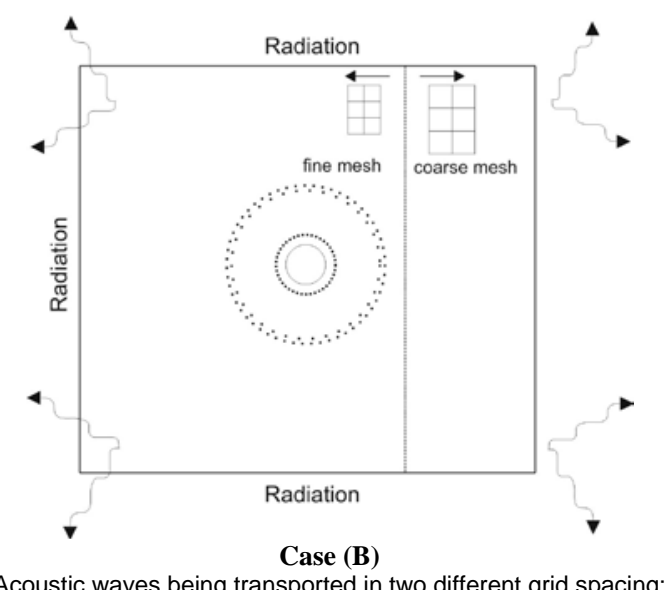

Figure 2. Illustration of wave propagation problems.

\section{Acoustic pulse inside a duct - CASE (C)}

In this case, it is considered the propagation of an acoustic pulse inside a duct in the presence of a mean flow of Mach number $\mathbf{M}=0.5$. The computational domain length is $-100 \leq x \leq 100$ and $-50 \leq y \leq 50$, where solid walls are located. A uniform grid of $\Delta x=\Delta y=1$ has been used. Two PML domains of width $10 \Delta x$ are included at either end of the open duct - Figure 3. 


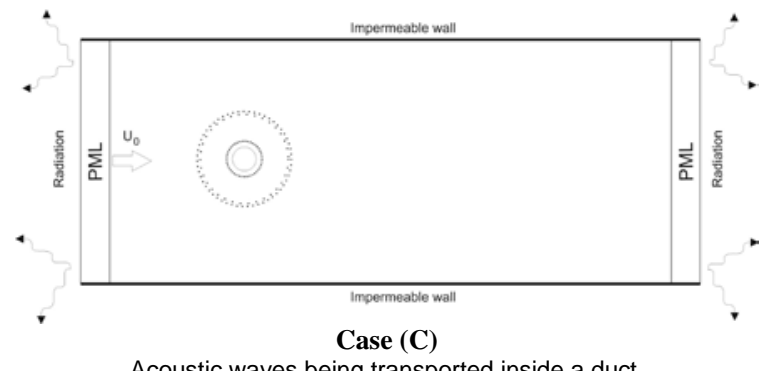

Figure 3. Illustration of wave propagation problems.

\section{RESULTS AND DISCUSSION}

In order to enhance the comprehension of the analysis performed in this work, the results will be separated in three sections according to the cases investigated.

\section{A. Numerical Results - CASE (A)}

Table 2 presents the performance results for the combination of the spatial and temporal schemes used in this work for solving the problem of Case (A). Observing the CPU time column, it's possible to see that the combination of RK3 and DRP7 scheme has the best performance. This result was expected since the RK3 scheme has less storage requirements by iteration. Moreover, it is important to note that the residual error associated with this scheme has the same order of magnitude when compared to the other combinations. However, it is noticeable that the optimized finite difference scheme of Bogey and Bailly (2002) showed the best performance at all, with the residual error decaying almost 1 order of magnitude. Besides, the increase in the computational cost seems to be acceptable. This, all the combinations studied in this work could be completely applied for CAA simulations.

Table 2. Performance of the numerical schemes CASE (A) investigation. $\mathrm{M}=0.5, \mathrm{CFL}=0.1$.

\begin{tabular}{|c|c|c|c|}
\hline \multicolumn{4}{|c|}{ DRP -Tam and Webb (1993) + Radiation \& Outflow Boundary Conditions } \\
\hline Runge-Kutta & LDD-FD scheme & CPU time (seconds) & $E_{R M S}$ \\
\hline RK3 & \multirow{3}{*}{ DRP 7-points } & 275.50 & $1.715 \times 10^{-7}$ \\
\hline LDDRK5 & & 422.39 & $1.741 \times 10^{-7}$ \\
\hline RKO5 & & 422.82 & $1.760 \times 10^{-7}$ \\
\hline \multicolumn{4}{|c|}{ FDo - Bogey and Bailly (2002) + Radiation \& Outflow Boundary Conditions } \\
\hline RK3 & \multirow{3}{*}{ STO 9-points } & 281.52 & $1.015 \times 10^{-7}$ \\
\hline LDDRK5 & & 435.93 & $9.641 \times 10^{-8}$ \\
\hline RKO5 & & 435.23 & $9.394 \times 10^{-8}$ \\
\hline \multicolumn{4}{|c|}{ DRP - Tam and Webb (1993) + Perfectly Matched Layer Boundary Conditions } \\
\hline LDDRK5 & DRP 7-points & 449.20 & $1.737 \times 10^{-7}$ \\
\hline
\end{tabular}

It is important to note that the LDDRK5+DRP7 combination for the PML method has a slightly higher computational cost when compared to the same combination when solving the outflow boundary conditions. It means, that the number of computations in the PML outer domain plays an important role in time consuming and that is cheaper to solve more equations in less points, as it is the case of the outflow boundary equations which are solved in 3 ghost's points.

Figure 4 shows the evolution of the residual error against the CFL number. As the CFL number goes to 0.5 (the maximum value used in this work) the error increases 1 order of magnitude, but it remains still within an acceptable range. During the analysis it was seen that the CFL can reach values up to 1.2 when using the PML technique.

Qualitative results, through contour plots, for the acoustic, entropy and vorticity wave propagation are shown in Figures 5, 6, 7 and 8. For brevity, only the pressure and entropy pulses are shown in these figures. The parameters used were intensity relative to peak disturbance pressure and density \pm 0.005 , $\pm 0.001, \pm 0.01, \pm 0.05$.

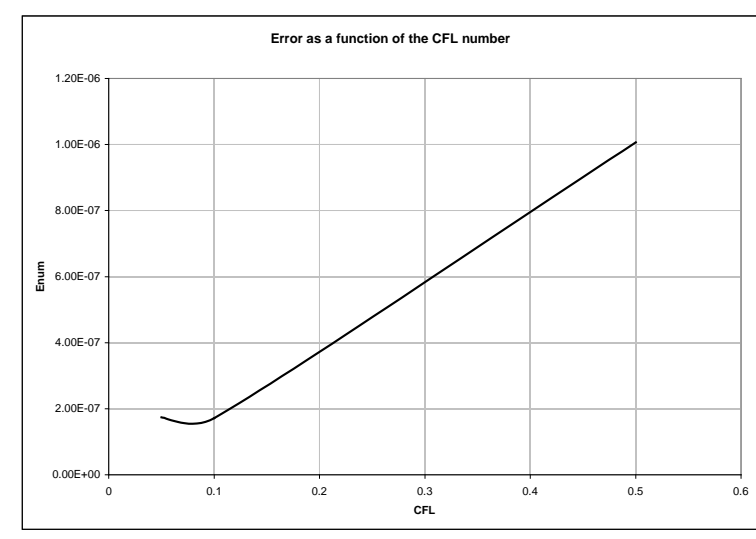

Figure 4. Residual error vs CFL number - Case (A) problem. LDDRK5+DRP7 (outflow boundary condition applied).

The pressure and density waveforms are also shown in these plots and compared with the exact solution - Equations (17), (18) and (19).

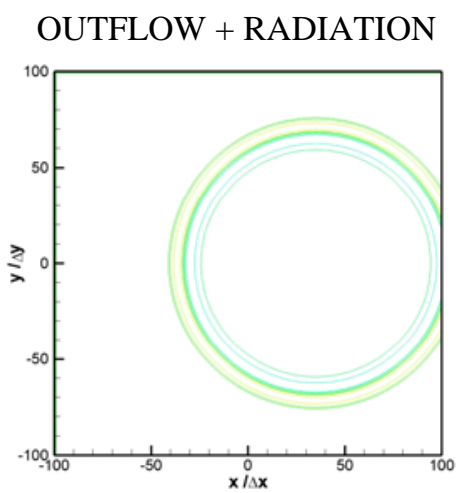

(a) Pressure Contour 


\section{Ciência/Science}

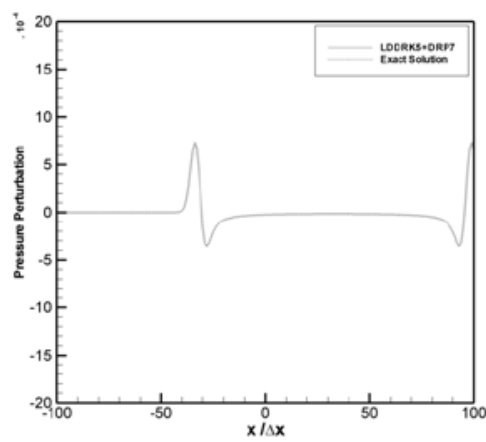

(b) Pressure waveform

Figure 5. Pressure contour and waveform LDDRK5+DRP7, M=0.5, CFL = 0.1.

The arrangement of releasing the pulses guarantees that the pressure and the entropy and vorticity waves are caught together at the same time in the right boundary of the domain - see Figure 5 and Figure 6 (only density waveform). In this situation the outflow boundary condition and the PML should allow the waves to leave the domain without considerable reflections levels. The waveforms plots and the residual error encountered in all simulations confirm that low level of reflection was found (used to be less than $1 \%$ of the incident wave amplitude). Therefore, both outflow and PML are transparent to the wave's propagation. An excellent agreement with the exact solution is seem in all combinations.

In order to verify the influence of the number of points in the PML zone, the absorption coefficient $\sigma \mathrm{m}$ and the exponent $\beta$ in the power function (15), three additional simulations were performed, varying the respective parameters. These results have been compared with the reference simulation used to compose the data shown in Table 2 and Figures 7 and 8 (only density waveform), which had 10 points in the PML zone and $\sigma \mathrm{m}=2$.

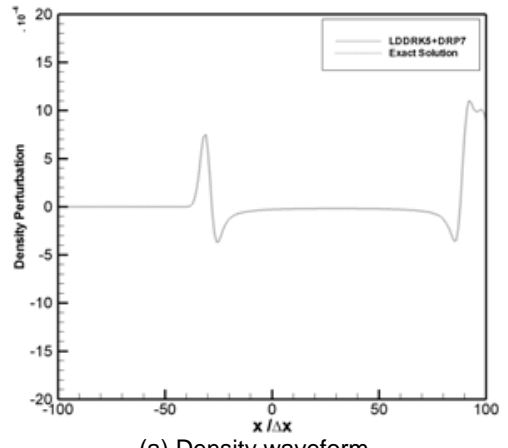

(a) Density waveform

Figure 6. Density waveform - LDDRK5+DRP7, $\mathrm{M}=0.5, \mathrm{CFL}=0.1$.

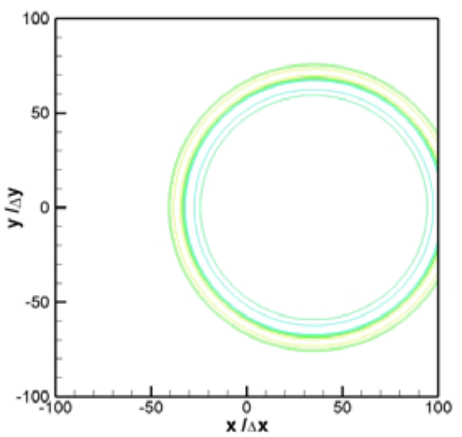

(a) Pressure Contour

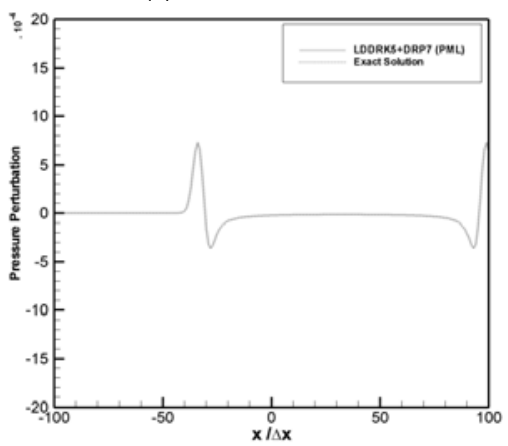

(b) Pressure waveform

Figure 7. Pressure contours and waveforms LDDRK5+DRP7, M=0.5, CFL $=0.1$.

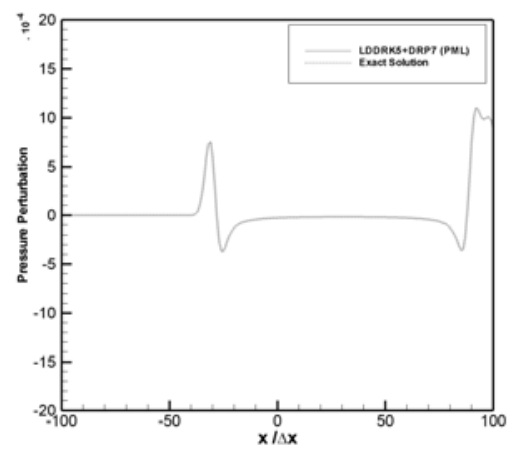

(a) Density waveform

Figure 8. Density waveform - LDDRK5+DRP7, $\mathrm{M}=0.5, \mathrm{CFL}=0.1$.

In the first simulation $\sigma \mathrm{m}$ was kept equal to 2 and the number of points in the PML zone was reduced to 5. Figure 9(a) presents the comparison of the pressure waveform at 800 time-steps against the exact solution.

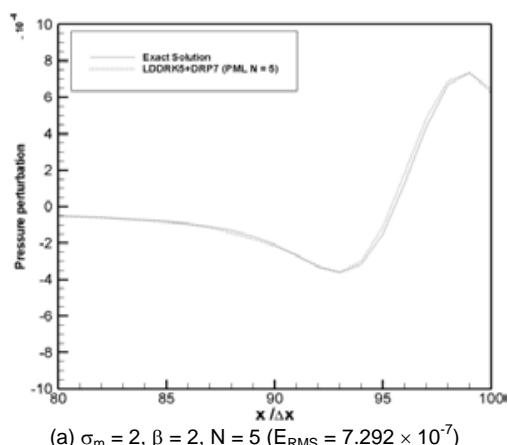




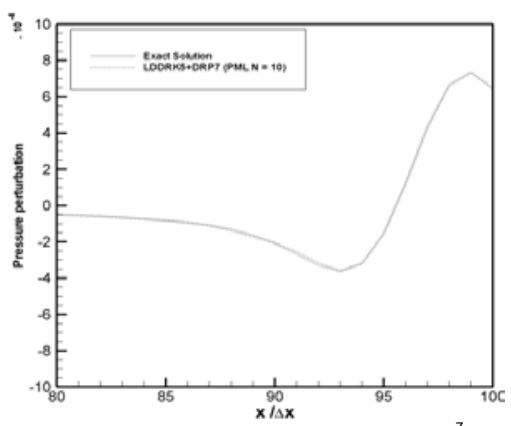

(b) $\sigma_{\mathrm{m}}=1.5, \beta=2, \mathrm{~N}=10\left(\mathrm{E}_{\mathrm{RMS}}=1.733 \times 10^{-7}\right)$

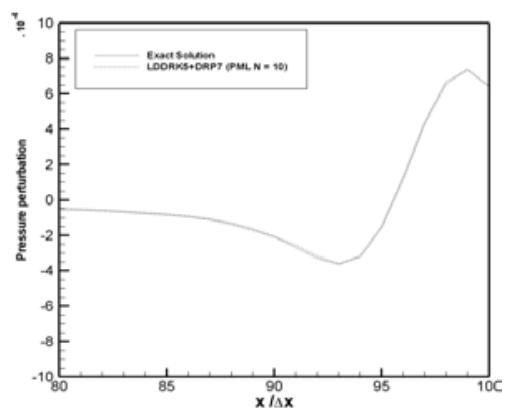

(c) $\sigma \mathrm{m}=2, \beta=1, \mathrm{~N}=10($ ERMS $=1.729 \times 10-7)$

Figure 9. Analysis of the influence of parameters in the PML power function absorption - Reference solution has a residual error ERMS $=1.737 \times 10-7$.

Note that the residual error increased 4 times when compared with that one showed on Table 2. However the boundary treatment still remains as transparent to the wave propagation. Figure 9(b) shows the results assuming $\sigma \mathrm{m}=1.5$ and keeping 10 points in the PML zone. Again, no noticeable impact is seemed since the error remains close to the reference simulation. The whole pattern of the solution is pretty the same as the reference solution. Finally, a more severe change was made with the exponent $\beta$, changing it to unity. By assuming that, it means that the absorption power function is now linear. The results are shown in Figure 9(c). It was interesting to observe that the final result reached the same order of residual error as presented by the reference solution. This quick analysis leads to the conclusion that the number of points in the PML zone is the most important parameter to deal in the absorption function.

\section{B. Numerical Results - CASE (B)}

The optimized schemes presented in this paper were originally based on a uniformly spaced grid. However, when there is stretching associated to the mesh significant discretization errors may occur, which may jeopardize the dispersion and dissipation properties inherent to these schemes. In order to verify the impact of non-uniform computational grid 3 different stretch rates were applied (5\%, 10\% and $50 \%)$. An acoustic pulse is propagated from an uniform mesh (region 1) to a new mesh (region 2) where it is applied the stretching. The interface between the two mesh resolutions is placed at $x / \Delta x=50$.

Figure 11 clearly shows the acoustic pulse close to the border of the computational domain. As the stretching rate is increased the acoustic pulse is distorted due to the spatial resolution. Consequently the wave front moves ahead, leading to a dispersion error, which in this simple case is relevant when compared to the exact solution - see Figure 10. Based on this simple analysis and in a literature review the recommended stretching rates cannot be more than $5 \%$.

CASE (B) - Effect of a non-uniform grid in the dispersion and dissipation properties.

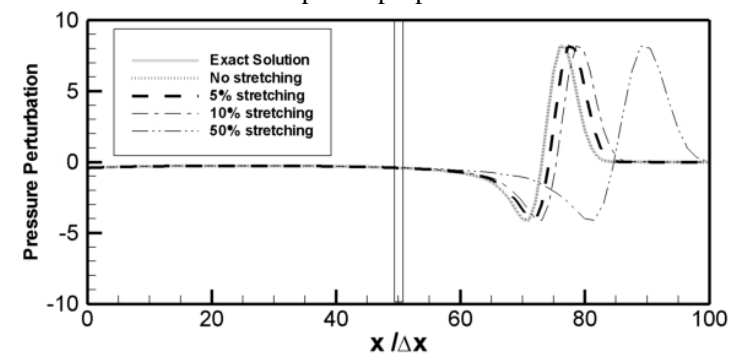

Figure 10. Effect of grid stretching over the acoustic pulse propagation - LDDRK5+DRP7, M=0.5, $\mathrm{CFL}=0.1$. The interface between the two mesh resolutions is marked with double-line.

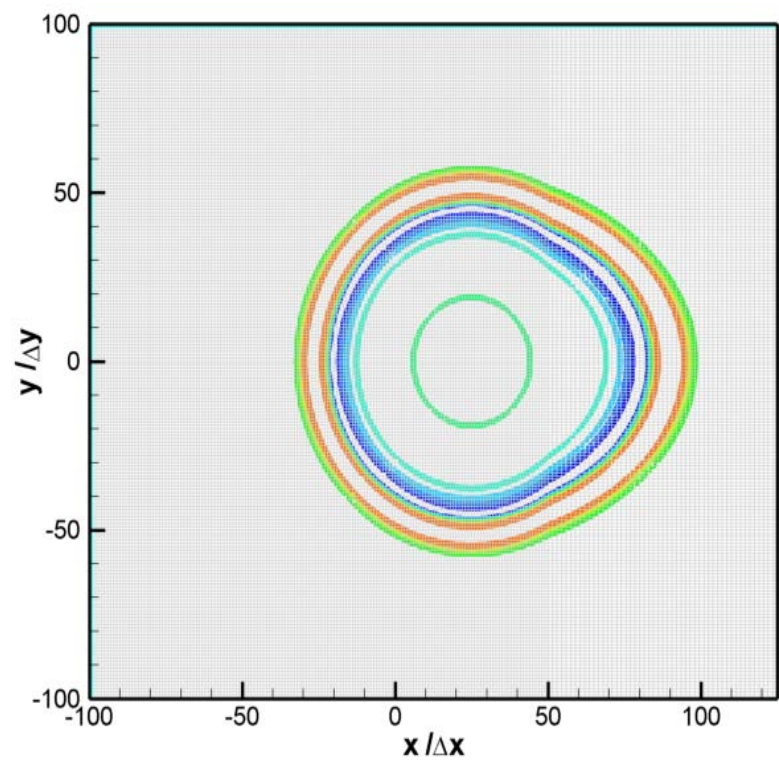

Figure 11 - Effect of grid stretching over the acoustic pulse propagation - LDDRK5+DRP7, M=0.5, $\mathrm{CFL}=0.1$. Pressure contours.

\section{Numerical Results - CASE (C)}

The last case studied was the propagation of an acoustic pulse inside a duct in the presence of a mean flow of Mach number $M=0.5$. Despite there is no available an analytical solution for this problem, the 
effectiveness of the boundary treatment could be extensively tested due to the strong incidence of waves that are leaving the domain and those ones that are being reflected by the wall and shall leave the domain as it is advected. The numerical scheme used was the LDDRK5+DRP7 keeping the CFL equal to 0.1 , for both set of radiation and PML boundary conditions. Figure 12 shows the pressure contours evolution inside the duct with time steps ranging from 200 up to 1400 varying in steps of 200. As the acoustic wave is advected inside the duct, it is reflected by the duct walls. Both set of boundary conditions (radiation and PML) could deal with the physical reflection of these waves. However, a close look on Figure 12 reveals that the PML treatment absorbs more the wave pattern's evolution. The pressure history was acquired in a point over the left border with coordinates of $(-97 ; 0)$ in order to confirm such fact. This data will not be showed here due to space limitations. The absence of an analytical solution does not permit to affirm which situation is closer to the actual physical of the problem. However, the "wiggles" seem in the borders of the radiation condition seems to be a signal that the solution is being contaminated by spurious reflections.

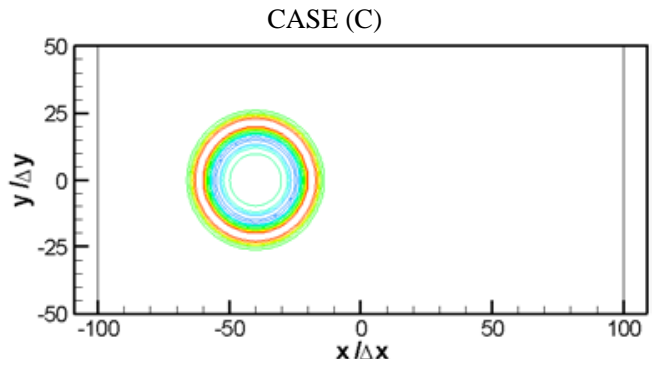

(a) $M=0.5$, time step $=200(P M L)$

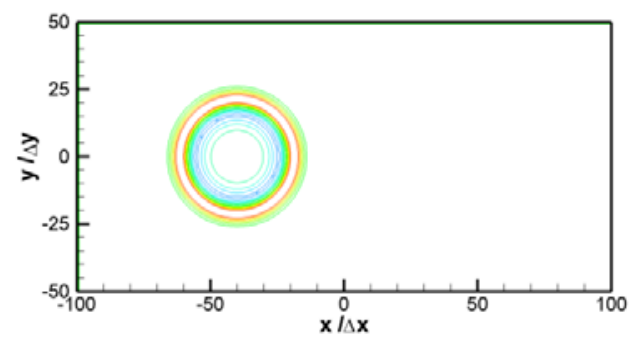

(a) $M=0.5$, time step $=200($ RADIATION $)$

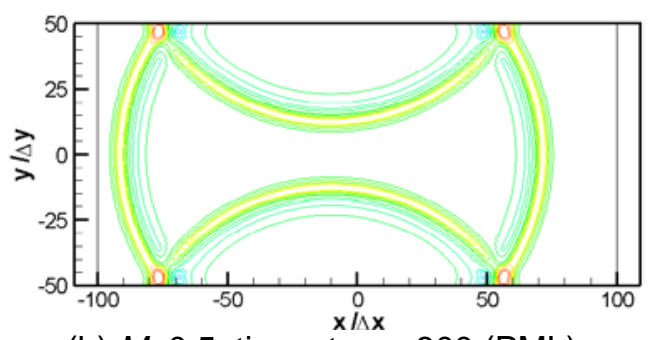

(b) $M=0.5$, time step $=800(P M L)$

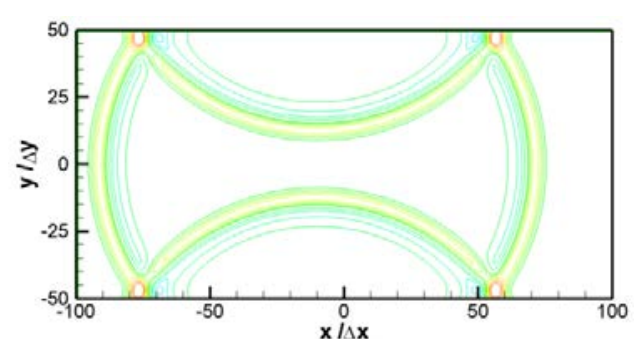

(b) $M=0.5$, time step $=800$ (RADIATION)

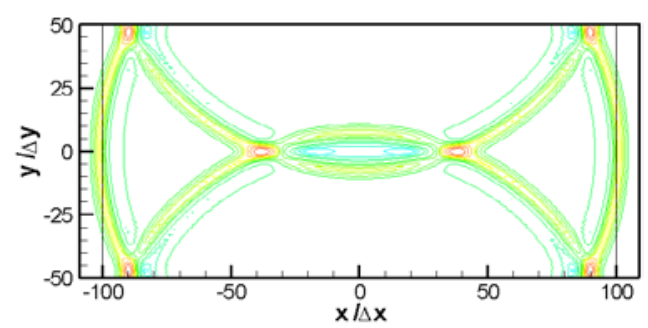

(c) $M=0.5$, time step $=1000(P M L)$

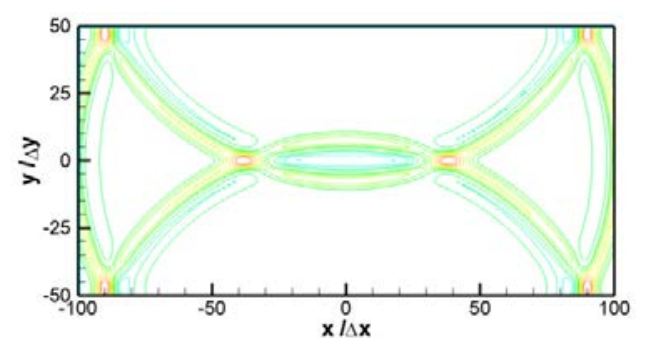

(c) $M=0.5$, time step $=1000($ RADIATION $)$

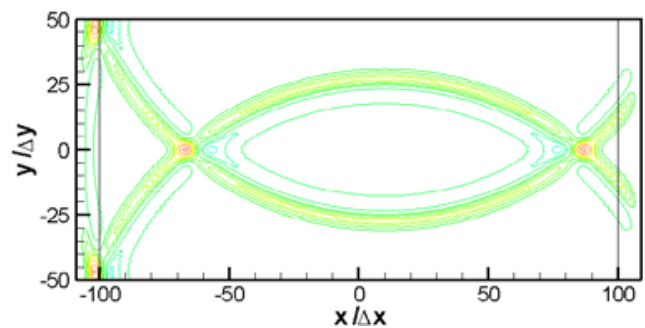

(d) $M=0.5$, time step $=1200(\mathrm{PML})$

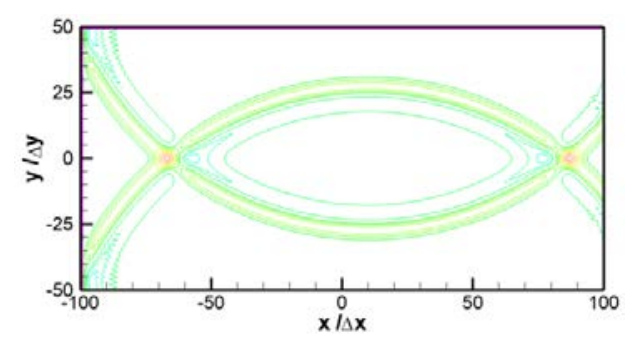

(d) $M=0.5$, time step $=1200$ (RADIATION)

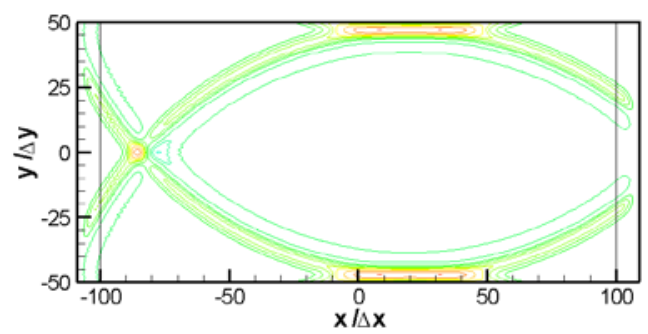

(e) $M=0.5$, time step $=1400(\mathrm{PML})$ 


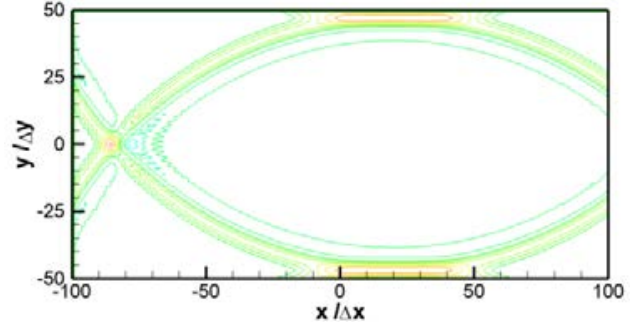

(e) $M=0.5$, time step $=1400$ (RADIATION)

Figure 12. Wave propagation inside a duct. Comparison of PML vs Radiation boundary treatment.

\section{CONCLUSIONS}

In this work a review about the performance of numerical schemes and boundary conditions for wave propagation problems was performed. Different spatial discretization as DRP and FDo as well as temporal Runge-Kutta schemes has been implemented in order to propagate pressure, entropy and vorticity disturbances throughout a 2D (twodimensional) domain. At the boundaries, radiation, outflow and perfectly matched layer equations were used to evacuate the pulses under the effect of a mean flow. With the numerical results presented herein, it was possible to identify which combination of the schemes studied better apply for solving such kind of wave propagation. For all simulations performed in the presence of a mean flow, the numerical schemes were stable and provided good results. Based on the results the recommended combinations are LDDRK5+DRP7 and RKO5+STO9, considering a trade between performance and accuracy. The boundary conditions tested were efficient in evacuating the pressure and entropy pulse perturbation with no noticeable reflections and instabilities in the computed waveforms.

\section{ACKNOWLEDGEMENTS}

The author would like to thank the "Fundação de Amparo à Pesquisa do Estado de Minas Gerais”FAPEMIG for the support.

\section{REFERENCES}

Bayliss, A., and Turkel, E., 1982, Far Field Boundary Conditions for Compressible Flows, Journal of Computational Physics, Vol. 48, pp. 182199.

Berland, J., Bogey, C., and Bailly, C., 2004, Optimized Explicit Schemes: Matching and Boundary Schemes and 4th-order Runge-Kutta algorithm, in: 10th AIAA/CEAS Aeroacoustics Conference, AIAA 2004-2814.

Berland, J., Bogey, C., and Bailly, C., 2005, Low-Dissipation and Low-Dispersion Fourth Order Runge-Kutta Algorithm, Computer and Fluids.

Bogey C., and Bailly, C., 2004, A Family of
Low-Dispersive and Low-Dissipative Explicit Schemes for Flow Noise and Noise Computations, Journal of Computational Physics, Vol. 194, pp. 194214.

Bogey C., and Bailly, C., 2002, A Family of low Dispersive and low Dissipative Explicit Schemes for Computing the Aerodynamic Noise, AIAA-paper 2509.

Colonius, T., Lele, S. K., and Moin, P., 1993, Boundary Conditions for Direct Computation of Aerodynamic Sound Generation, AIAA Journal, Vol. 31, No. 9, pp. 1574-1582.

De Roeck W., Desmet W., Baelmans M., and Sas P., 2004, An Overview of High-Order Finite Difference Schemes for Computational Aeroacoustics, in: International Conference on Noise \& Vibration Engineering, pp. 353-368.

Giles, M. B., 1990, Nonreflecting Boundary Conditions for Euler Equation Calculations, AIAA Journal, Vol. 28, No. 12, pp. 2050-2058.

Givoli, D., 1991, Non-Reflecting Boundary Conditions, Journal of Computational Physics, Vol. 94, pp. 1-29.

Haras, Z., and Ta'asan, S., 1994, Finite Difference Schemes for Long-Time Integration, Journal of Computational Physics, Vol. 114, pp. 265.

Hu, F. Q., Hussaini, M. Y., and Manthey, J. L., 1996, Low-Dissipation and Low-Dispersion RungeKutta Schemes for Computational Acoustics, Journal of Computational Physics, Vol. 124, pp. 177-191,

Hu, F., Q., and Atkins, H. L., 2002, Eigensolutions Analysis of the Discontinuous Galerkin Method with Nonuniform Grids. Part I: one Space Dimension, Journal of Computational Physics, Vol. 182, pp. 516-545.

Hu, F. Q., 1996a, On Absorbing Boundary Conditions of Linearized Euler Equations by a Perfectly Matched Layer, Journal of Computational Physics, Vol. 129, pp. 201-219.

Hu, F. Q., 1996b, On Perfectly Matched Layer as an Absorbing Boundary Condition, AIAA paper 96-1664.

Hu, F. Q., 2001, A Stable, Perfectly Matched Layer for Linearized Euler Equations in Unsplit Physical Variables, Journal of Computational Physics, Vol. 173, pp. 455-479.

Hu, F. Q., 2002, On Constructing Stable Perfectly Matched Layers as an Absorbing Boundary Condition for Euler Equations, AIAA paper 20020227.

Israeli, M., and Orszag, S. A., 1981, Approximation of Radiation Boundary Conditions, Journal of Computational Physics, Vol. 41, pp. 115135.

Lele, S. K., 1992, Compact Finite Difference Schemes with Spectral-Like Resolution, Journal of Computational Physics, pp. 16-42.

Lockard, D. P., Brentner, K. S., and Atkins, H. L., 1995, High-Accuracy Algorithms for Computational Aeroacoustics, AIAA Journal, Vol. 33, No. 2, pp. 246. 
Rai, M. M., and Moin, P., 1991, Direct Numerical Simulation of Transition and Turbulence in a Spatially Evolving Boundary Layer, AIAA paper 91-1607.

Stanescu, D., and Habashi, W. G., 1998, 2NStorage low Dissipation and Dispersion Runge-Kutta for Computational Acoustics, Journal of Computational Physics, Vol. 143, pp. 674-681.

Ta'asan, S., and Nark, D. M., 1995, An Absorbing Buffer Zone Technique for Acoustic Wave Propagation, AIAA paper 95-0164.

Tam, C. K. W., and Dong, Z., 1994, Radiation Boundary Condition and Anisotropy Correction for Finite Difference Solutions of the Helmholtz Equation, Journal of Computational Physics, Vol. 113, pp. 122-133.

Tam, C. K. W., and Webb, J. C., 1993, Dispersion-Relation-Preserving Finite Difference Schemes for Computational Acoustics, Journal of Computational Physics, Vol. 107, pp. 262-281.

Tam, C. K., 1995, Computational Aeroacoustics: Issues and Methods, AIAA Journal, Vol. 33, pp. 10.

Tam, C. K., 1997, Advances in Numerical Boundary Conditions for Computational Aeroacoustics, American Institute of Aeronautics and Astronautics, AIAA paper-1774, pp. 1-16.

Tam, C. K., 2004, Computational aeroacoustics: An Overview of Computational Challenges and Applications, International Journal of Computational Fluid Dynamics, Vol. 18, No. 6, pp. 547-567.

Williamson, J. H., 1980, Low-Storage RungeKutta Schemes, Journal of Computational Physics, Vol. 35, pp. 48-56.

Zingg, D. W., Lomax, H., and Jurgens, H., 1993, An Optimized Finite Difference Scheme for Wave Propagation Problems, AIAA paper 93-0459. 